\title{
Pulsatile Arterial Bleed from Uretero-Vesical Junction following Placement of a Double J Stent: An Endo-Urology Nightmare
}

\author{
Samarth Agarwal ${ }^{1}$, Ashish Sharma ${ }^{*}$, Akansha Gupta ${ }^{2}$, Anamika Sharma ${ }^{3}$, Siddharth Pandey ${ }^{1}$, Deepanshu \\ Sharma ${ }^{1}$ and Satya Narayan Sankhwar ${ }^{1}$
}

${ }^{1}$ Department of Urology, King George's Medical University, India

${ }^{2}$ Department of obstetrics and gynaecology, Jolly Grant Medical College, India

${ }^{3}$ Department of Anesthesia and Critical Care, All India Institute of Medical Sciences, India

Submission: July 13, 2018; Published: August 10, 2018

*Corresponding author: Ashish Sharma, Senior Resident (MCh Urology), Department of Urology, King George's Medical University (KGMU), Pin: 226003, India, Email: ashishuc343@gmail.com

\section{Abstract}

The ureteric stent placement is amongst the commonest urological procedures undertaken for urological diseases. It is indicated for relief of obstructive uropathy as a temporary procedure or after endourological surgeries like ureteroscopy, stone surgery, pyeloplasty etc. Various complications which may be associated with ureteric stenting are irritative voiding symptoms (50-60\%), urgency (57-60\%), dysuria (40\%), incomplete emptying (76\%), incontinence, and haematuria (25\%). Haematuria is particularly worrisome complication and needs special emphasis. We hereby report a case of massive haematuria seen after DJ stent placement which was managed with cystoscopy clot evacuation followed by cauterization of active bleeder with bugbee electrodes application. The urologists, especially trainees, need to be aware of this dreaded complication and its management.

Keywords: DJ Stent; Haematuria; Endourology; Supravesical obstruction; Lithotripsy

\section{Introduction}

Ureteral stenting is widely used urological procedure to overcome supravesical obstruction or as an adjunct with various elective urological surgeries like pyeloplasty, pyelolithotomy, percutaneous nephrolithotomy, ureteroscopy lithotripsy and ureteric preimplant. The complications of double J (DJ) stenting are observed more frequently with the increased use of ureteric stents. Early complications of DJ stents are stent related discomfort, irritative voiding symptoms and bleeding. In contrast, late complications of stenting are encrustation, migration, blockage and fragmentation. Haematuria is particularly worrisome complication and needs special emphasis. Massive haematuria after stent placement is a rare and life-threatening complication. We hereby report a case of massive haematuria seen after DJ stent placement which was managed with cystoscopy clot evacuation followed by cauterization of active bleeder with Bugbee electrodes application. The urologists, especially trainees, need to be aware of this dreaded complication and its management.

\section{Case Report}

A 40yr old female patient was referred to out-patient department with complaints of right flank pain for last 15 days. On clinical evaluation she was afebrile with normal blood pressure.
Her abdominal examination was unremarkable. Her complete blood hemogram was normal except elevated serum creatinine - $3.14 \mathrm{mg} / \mathrm{dl}$. On evaluation with ultrasound KUB (kidney ureter bladder) she was found to have enlarged Right kidney with Hydroureteronephrosis with right mid ureteric calculus and left small Atrophic kidney. On further evaluation with NCCT-KUB she was found to have Right moderate hydroureteronephrosis with mid ureteric calculi of size $10 \mathrm{~mm}$ and left renal agenesis.

A 5F double J (DJ) stent was placed under Local Anaesthesia with full antibiotic coverage and aseptic precautions. The patient was planned for uretero-reno scopic (URS) removal of calculi at a later date. However, she referred back after 4 days with anuria and clot retention for last $12 \mathrm{hrs}$ with serum creatinine of $6.5 \mathrm{mg} / \mathrm{dl}$. Her Hb was 7.5gm/dl, TLC 13000 . Blood coagulation parameters like Prothrombin time, activated partial thromboplastin time and INR were also normal. She underwent cystourethroscopy with clot evacuation elsewhere 2 days back.

On examination her bladder was palpable with a suprapubic lump. On repeat cystourethroscopy performed under intravenous sedation, the urinary bladder was found to be distended and full of blood clots. Once complete clot evacuation was done meticulously, a pulsatile bleed was identified from Right Ureter 
vesical junction as shown in the (Figure 1). This pulsatile bleed was coagulated with a Bugbee electrode as shown in (Figure 2). Following this procedure, her condition improved drastically in next $24 \mathrm{hrs}$. Her creatinine had fallen to $1.3 \mathrm{mg} / \mathrm{dl}$ and there was no recurrence of haematuria.
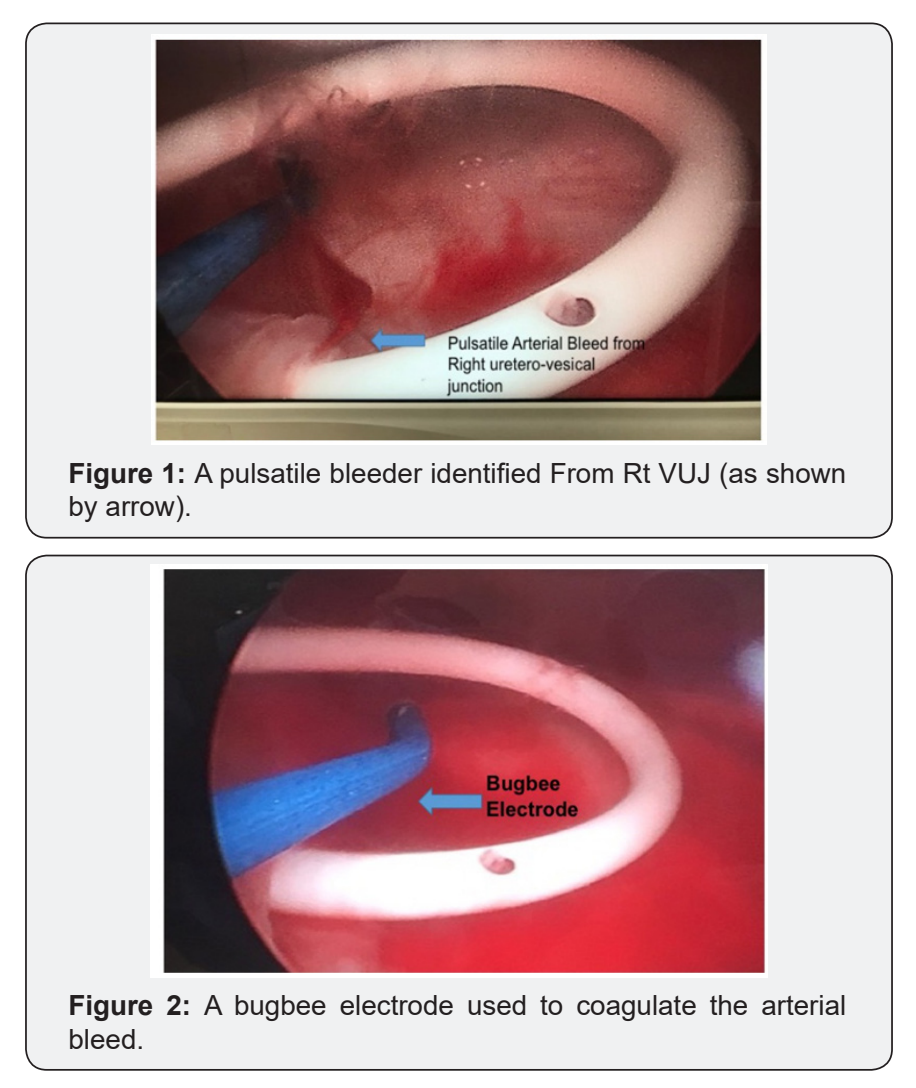

\section{Discussion}

First describe by Zimskind in 1967, placement of a double J stent is amongst the commonest urological procedures undertaken. It is amongst the first procedure performed by a urology trainess resident and is an indispensable tool for urologist armamentarium. Consequential indications of Double J stent are plenty and include intolerable renal colic, obstructive pyelonephritis, stein Strasse, history of Renal Failure and solitary or transplant kidney. [1]

Although being overused in clinical practice, it is associated with fair share of complications. Several studies in literature describe the symptoms related to ureteral stents and their respective incidence: irritative voiding symptoms (50-60\%), urgency (57-60\%), dysuria (40\%), incomplete emptying (76\%), incontinence, and haematuria (25\%).

Haematuria is particularly worrisome as reported by Ho Ch et al. [2]. The exact aetiology underlying haematuria following stenting is not elucidated. In our case, it might have been due to stretching of ureter vesical orifice while placement of stent leading to tearing of the underlying vessel. Urologists, especially trainees, need to be aware of this dreaded complication.

\section{Conclusion}

a) The DJ stent placement is amongst the first urological procedure undertaken by a resident trainee.

b) Haematuria following stent placement is a common complication- in severe cases patient may resent with clot retention and anuria.

c) There is some amount of stretching of ureter vesical junction while placing a $5 \mathrm{Fr}$ stent which might result in rupture of underlying vessel.

d) Once diagnosed, such bleeding can be easily controlled by endoscopic intervention. Urologists, especially trainees, need to be aware of this dreaded complication.

\section{References}

1. Jeong H, Hwak C, Lee SE (2004) Ureteric stenting after ureteroscopy for ureteric stones: a prospective randomized study assessing symptoms and complications. BJU Int 93(7): 1032-1035.

2. Ho CH, Chen SC, Chung SD, Lee YJ, Chen J, et al. (2008) Determining the Apropriate Length of a Double-Pigtail Ureteral Stent by Both Stent Configurations and Related Symptoms. J Endourol 22(7): 1427-1431.

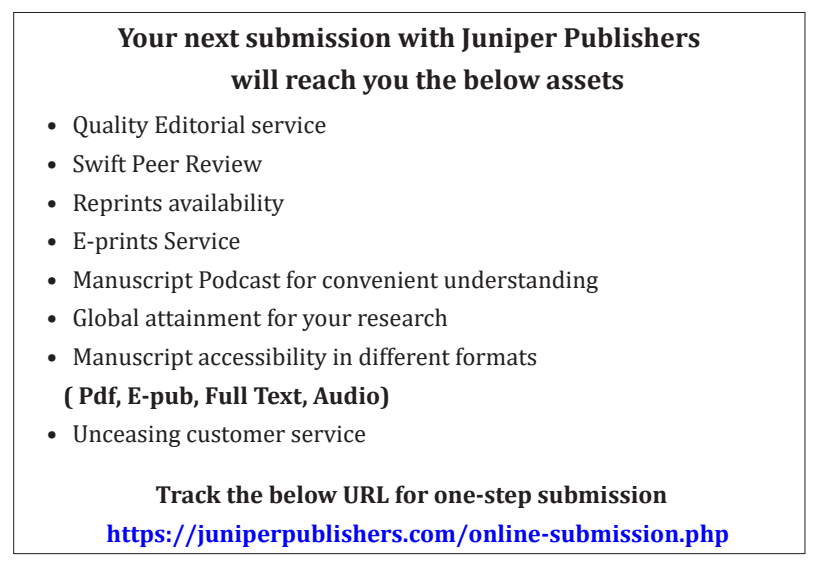

